\title{
Esquemas mentales desadaptativos según la composición familiar en adolescentes arequipeños de escuelas públicas
}

\author{
Disadaptive mental schemas according to family composition in Arequipa \\ adolescents of public schools
}

\author{
Rosa Angélica Seperak Viera ${ }^{1}$ \\ Luz Marina Díaz Huayna ${ }^{2}$ \\ María Daniela Canazas Bustios ${ }^{3}$ \\ Salomón Shelach Bellido ${ }^{4}$ \\ Universidad Católica San Pablo
}

Recibido: $15-10-18$

Aceptado: $27-12-18$

\begin{abstract}
Resumen
El impacto de la familia en el desarrollo del individuo ha sido objeto de muchas investigaciones, más aún por la variabilidad estructural en la que se encuentra inmersa y que ha cobrado protagonismo en el análisis de la salud psicológica de los miembros que la componen. El presente estudio tuvo como objetivo realizar un análisis de los esquemas mentales desadaptativos según el tipo de estructura familiar de adolescentes de 13 escuelas públicas de Arequipa Metropolitana. Se utilizó como instrumento el Cuestionario de Esquemas de Young (YSQ-L2) el cual fue validado en Perú por León y Sucari (2012). Los hallazgos demuestran que existen diferencias entre los hogares reconstruidos y las demás estructuras en cuanto a la presencia de los esquemas mentales desadpatativos de privación emocional, desconfianza, hipercritinismo y grandiosidad.
\end{abstract}

Palabras clave: Esquemas mentales desadaptativos; Jeffrey Young; composición familiar.

\begin{abstract}
The impact of the family on the development of the individual has been the subject of much research, especially because of the structural variability in which it can be immersed. Moreover, it has gained prominence in the analysis of the psychological health of its members. The objective of this study was to perform an analysis of the maladaptive mental schemes according to the type of family structure in adolescents of 13 public schools in Metropolitan area of Arequipa. The Young Schema Questionnaire (YSQ-L2) was used as an instrument, which was validated in Perú by León and Sucari (2012). The results shows that there are differences between reconstructed homes and other structures in terms of the presence of maladaptive mental schemes of emotional deprivation, distrust, hypercritism and grandiosity.

Keywords: Maladaptive mental schemes; Jeffrey Young; family structure.
\end{abstract}

1 Docente,Universidad Católica San Pablo. Autor para correspondencia. E-mail: raseperak@ucsp.edu.pe

2 Bachiller,Universidad Católica San Pablo. E-mail: luz.diaz@ucsp.edu.pe

3 Bachiller,Universidad Católica San Pablo. E-mail: maria.canazas@ucsp.edu.pe

4 Estudiante, Universidad Católica San Pablo. E-mail: salomon.shelach@ucsp.edu.pe

(C) Los autores. Este artículo es publicado por la Revista de Investigación en Psicología de la Facultad de Psicología, Universidad Nacional Mayor de San Marcos. Este es un artículo de acceso abierto, distribuido bajo los términos de la licencia Creative Commons Atribucion - No Comercia_Compartir Igual 4.0 Internacional. (http://creativecommons.org/licenses/by-nc-sa/4.0/) que permite el uso no comercial, distribución y reproducción en cualquier medio, siempre que la obra original sea debidamente citada. 


\section{INTRODUCCIÓN}

La teoría de esquemas mentales desadaptativos es una ampliación de la teoría cognitiva conductual y combina elementos de la teoríapsicoanalista, constructivista, gestalt, del apego y relaciones objetales (Young, Klosko \& Weishaar 2013). El concepto de esquema mental está definido como una estructura cognitiva que adquiere el individuo para darle significado a los eventos más significativos dentro de su entorno (Ferrel, Peña, Gómez \& Pérez, 2009; Young et al., 2013).

Los esquemas mentales desadaptativos (EMD) son estructuras impuestas sobre la realidad, que ayuda a las personas a explicar y orientar sus respuestas según cada acontecimiento vivido (Young et al., 2013). Conservan un carácter egodistónico, son contraproducentes y si no son tratados a tiempo se solidifican en la adultez y se convierten en el núcleo de los trastornos de personalidad (Rodríguez, 2009; Lemos, Londoño \& Zapata, 2007; Castrillón, Chávez, Ferrer, Londoño, Maestre, Marín \& Schniter, 2005).

Los EMD son patrones que carecen de flexibilidad, variabilidad; creatividad y limitan la vida de la persona ya que contemplan necesidades no cubiertas en la infancia (Vite, Negrete, \& Miranda, 2012). Se centran en necesidades emocionales básicas que debe percibir todo individuo en sus relaciones significativas básicamente dentro del contexto familiar (Rodríguez, 2009). En este sentido, las experiencias negativas, desbordantes y ausentes en las relaciones familiares funcionarían en la mayoría de los casos como uno de los factores etiológico en el desarrollo de los EMD (Mayorga, Godoy, Riquelme \& Ketterer, 2016; Gantiva, Bello, Vanegas \& Sastoque, 2009; Cabrera, Guevara \& Barrera, 2006).

La teoría de esquemas mentales desadaptativos ha sido plasmada de forma predominante en investigaciones que tienen como objeto de estudio al adolescente y factores de riesgo como depresión y ansiedad (León, Salazar \& Puerta, 2013); problemas sexuales (Cepeda \& Ruiz, 2016); trastornos alimentarios (De la Vega \& Gómez, 2012), problemas académicos (Bahamón, 2013), consumo de sustancias (Martínez, Robles, Utria \& Amar, 2014;), trastornos dismórficos corporales (Múnera \& Tamayo, 2013), problemas de conducta (Castro, López \& Sueiro, 2009) y para trastornos egodistónicos moldeados en la infancia (Estévez \& Calvete, 2007).

Jeffrey Young (Young et al., 2013), expone argumentos importantes sobre la causa de los EMD, lo que lo que condujo a plantear que las experiencias negativas del niño dentro del contexto familiar cobraban influencia en el desarrollo de estos patrones. Diversos autores comparten el mismo argumento indicando que la presencia de padres abusadores y/o sobreprotectores; la carencia de afecto y aislamiento; un estilo de crianza indulgente o autoritario y límites difusos configurarían una importante predominancia en el origen de los EMD. (Múnera \& Tamayo, 2013; Rodríguez, 2009). 
Ante lo expuesto, Bronfenbrenner (1979) considera que la familia es el sistema que configura en mayor grado a la persona y aporta en su evolución desde que se la concibe. A su vez el enfoque psicológico destaca a la familia como un todo y que el cambio en una de sus unidades cambia el todo (Valladares, 2008).

En la teoría sistémica formulada por Bertanlanffy (1968) se concibe a la familia dentro del microsistema más inmediato e influyente por los lazos y relaciones que se forma. Dentro del microsistema familia, se habla de diferentes estructuras o subsistemas, dentro de los cuales el subsistema conyugal/paternal y fraternal son los que representan mayor relevancia por su función de conservar la homeostasis y de esta forma procurar un bienestar integral a todos los miembros que la componen (Coleman, 2013; Roca, 2010; Belsky, 2005).

Parsons y Bales (1955), muestra a la familia como un sistema en el cual hay componentes que regulan las relaciones de interacción y jerarquía, los cambios bruscos en el microsistema o ausencia de los elementos necesarios para su funcionalidad entre subsistemas, traerían consigo problemas interpersonales e individuales, entre ellos los trastornos de personalidad (Cano, Motta, Valderrama \& Gil, 2016; Brown, Maning \& Payna, 2016; Perea \& Jiménez, 2008; Cavanagh, Crissey \& Raley, 2008). La multiplicidad o ausencia de algunos de los miembros de los subsistemas tendrían carácter de familia incompleta; sin embargo, lo que destaca la teoría sistémica es que se puede encontrar familias desequilibradas y disfuncionales desde una perspectiva de normalidad unidos por patrones de comportamiento y pensamiento que los unifica, pero que pueden constituir en algunos sentidos un desorden al sistema social y a los miembros que la componen (Coleman, 2013; Bárcenas, 2010; Valladares, 2008).

La amplia variedad de modelos familiares en la actualidad es manifestada a través de la ausencia de alguno de los padres como en los hogares monoparentales; en la disminución de la natalidad, que reduce los subsistemas fraternales; en el involucramiento de figuras paternales no biológicas como en las familias reconstituidas y la configuración de hogares sin figuras paternales ni fraternales como los hogares sin núcleo (Pliego \& Castro, 2015; Huarcaya, 2011; Roca, 2010). Esta diversificación en los hogares, multiplicidad y eliminación de algunos subsistemas y la nueva forma de funcionalidad, constituyen uno de los horizontes para la visualización de diferentes modos de pensamiento y comportamiento en las nuevas generaciones (Bárcenas, 2012, 2010; Rodríguez \& Menéndez, 2003; Shapiro, Gottman \& Carrére, 2000) a los que Palacios y Rodrigo (1998) llaman proceso de deconstrucción. Estos cambios por ser cada vez más rápidos y lejanos al núcleo natural, generan poca claridad sobre cómo deben impartirse los roles y funciones (Estévez \& Calvete, 2007), puesto que el subsistema conyugal/paternal cada vez es más confuso y diverso (Martínez, Estévez \& Inglés, 2013, Espinal et al., 2006; Rodríguez \& Menéndez, 2003). 
Los cambios estructurales en la familia son un resultado de la influencia de las fuerzas sociales, culturales, políticas, económicas e ideológicas ha ocasionado notables variantes al núcleo tradicional. (Pliego \& Castro, 2015; Bárcenas, 2010; Del Valle, 2004). Entre las fuerzas más significativas se encuentran la inserción de la mujer al mundo laboral (Huarcaya, 2011; Rodríguez \& Menéndez, 2003), el incremento de los divorcios (Cantón, Cortés \& Justicia, 2002), las diferencias en los roles en la crianza (Rogers \& White 1998), cambios en los estilos de vida (Stock, Corlyon, Castellanos \& Gieve, 2014), diferencias en las relaciones entre padres e hijos (Vite et al., 2012; Cabrera et al., 2006; Del Río, Barrera \& Vargas, 2004).

Desde una perspectiva estructural, estudios han evidenciado que son los hogares nucleares biparentales aquellos que contribuyen a una sana salud psicológica de todos los miembros que la componen (World Family Map, 2017; Pliego \& Castro, 2015; Observatorio de la Deuda Social Argentina, 2014; Cortés, Cantón \& Cantón, 2011; Huarcaya, 2011); los hijos provenientes de hogares monoparentales con jefatura femenina tienden a conductas agresivas y consumo de sustancias (Pera \& Jiménez, 2009), en los hogares de padres divorciados y con problemas de funcionalidad se ha encontrado mayores problemas de conducta en varones (Mayorga et al., 2016; Cantón, Cortés \& Justicia, 2002) y mayor predominancia de esquemas mentales disfuncionales en mujeres (Gantiva et al., 2009).

La intervención de la figura paterna en el proceso de apego y formación de los hijos pone de manifiesto la necesidad de concebir hogares intactos (Cuddeback, 2017; Cano et al., 2016; Olhaberry \& Santelices 2013; Perea \& Jiménez, 2009; Shapiro et al., 2000), calificando positivamente a las familias tradicionales por proporcionar un mejor ajuste psicológico a los hijos (Wilcox, 2017; Coleman, 2013; Cavanagh et al., 2008; Del Valle, 2004), a diferencia de las familias de padres que cohabitan (World Family map, 2017; Brown, Mainning \& Payne, 2016) y monoparentales (Wilcox, 2017; Pliego \& Castro, 2015; Olhaberry \& Farkas, 2012) consideradas como escenarios familiares próximos a la vulnerabilidad psicológica, social y económica.

\section{METODOLOGÍA}

\section{Tipo de Estudio}

El presente estudio es de corte cuantitativo debido a que las variables que se utilizaron fueron de magnitud numérica y los objetivos de nuestra investigación se respondían a partir de un trato estadístico que se le dio a nuestras variables de magnitud numérica. Cuenta con un diseño descriptivo porque busca especificar propiedades, características y rasgos dentro de la investigación; correlacional debido a que se busca encontrar la asociación de las variables en base al instrumento utilizado y transversal ya que la evaluación de la muestra se hizo en un solo momento (Hernández, Fernández y Baptista, 2014). 


\section{Participantes}

En el presente estudio contó con una muestra aleatoria de 726 estudiantes de escuelas públicas de 13 distritos de Arequipa Metropolitana en base a un nivel de confianza del $95 \%$ y un margen de error del 3.5\%. Los datos del universo de la muestra se encuentran registrados en el Portal de la Unidad de Estadística Educativa (ESCALE, 2017) del Ministerio de Educación del Perú (MINEDU, 2017), en el que se contabilizó 6560 estudiantes de quinto de secundaria de escuelas públicas de los 13 distritos especificados en el cuadro $\mathrm{N}^{\circ} 1$ hasta el año 2016. El número de estudiantes que participaron por colegio fue seleccionado en proporción de la cantidad de estudiantes registrada por distrito. La elección de las institución se llevó a cabo mediante un muestreo aleatorio estratificado (Hernández et al., 2014).

Tabla 1

Selección de la muestra según distrito

\begin{tabular}{lccc}
\hline Distrito & $\begin{array}{c}\text { No de colegios } \\
\text { estatales secundaria }\end{array}$ & $\begin{array}{c}\text { Alumnos de 5to } \\
\text { secundaria }\end{array}$ & $\begin{array}{c}\text { Proporción de } \\
\text { estudiantes por } \\
\text { colegio }\end{array}$ \\
\hline Cercado de Arequipa & 10 & 1082 & 116 \\
Cerro colorado & 16 & 1097 & 117 \\
Cayma & 9 & 674 & 72 \\
Yanahuara & 2 & 106 & 11 \\
Alto selva alegre & 8 & 534 & 57 \\
Miraflores & 4 & 291 & 31 \\
Mariano melgar & 6 & 670 & 71 \\
José Luis Bustamante y Rivero & 6 & 469 & 50 \\
Hunter & 13 & 352 & 37 \\
Paucarpata & 6 & 578 & 62 \\
Sachaca & 4 & 164 & 18 \\
Socabaya & 12 & 593 & 63 \\
Tiabaya & 3 & 193 & 21 \\
Total & 99 & 6560 & 726 \\
\hline
\end{tabular}

La muestra estuvo conformada por 308 varones (42.4\%) y 418 mujeres (57.6\%); considerando la composición o estructura familiar 349 de los estudiantes pertenecen a hogares nucleares biparentales $(48,1 \%) ; 179$ estudiantes a hogares monoparentales (24.7\%); 93 estudiantes a hogares extensos (12.8\%) y 42 estudiantes a hogares reconstruidos (5.8\%). 
Tabla 2

Sexo de la muestra

\begin{tabular}{lcc}
\hline & Frecuencia & Porcentaje \\
\hline Masculino & 308 & $42.40 \%$ \\
Femenino & 418 & $57.60 \%$ \\
Total & 726 & $100.00 \%$ \\
\hline
\end{tabular}

Tabla 3

Estructura familiar de la muestra

\begin{tabular}{lcc}
\hline & Frecuencia & Porcentaje \\
\hline Biparental & 351 & $48.30 \%$ \\
Extensa & 95 & $13.08 \%$ \\
Reconstruida & 44 & $6.06 \%$ \\
Monoparental & 181 & $24.93 \%$ \\
Sin Núcleo & 55 & $7.58 \%$ \\
Total & 726 & $99.9 \%$ \\
\hline
\end{tabular}

\section{Procedimiento}

Se estableció contacto con los directores de cada institución educativa, a los cuales se les explico los objetivos, importancia y procedimiento de la investigación. Al obtener la aprobación de las autoridades y el consentimiento informado firmado por los padres de los alumnos; los evaluadores coordinaron las fechas y horarios para la evaluación.

La resolución de la prueba tuvo una duración de 15 minutos por salón de forma colectiva; en la cual se hizo una breve explicación a los estudiantes indicando que los que trajeron el consentimiento informado firmado por los padres podrían participar de forma voluntaria en la evaluación, a su vez de la confidencialidad de los datos personales y se absolvieron sus dudas.

Se procedió al análisis de datos en el programa estadístico SPSS 24.0 (IBM, 2013). En el mismo se procedió a un análisis de normalidad de la muestra para analizar si la estadística a utilizar es paramétrica o no, luego de ello se hizo el análisis de validez y confiabilidad de la prueba para probar el uso del instrumento en la población a evaluar y por último se hizo el análisis descriptivo e inferencial de la muestra siguiendo los objetivos propuestos de la investigación.

\section{Instrumentos}

Variables sociodemográficas Se realizó una encuesta de preguntas relacionadas al sexo, edad, la estructura familiar (nuclear biparental, monoparental masculino y femenina, reconstruida, extensa y sin núcleo). 
Variable esquemas mentales desadaptativos Young (1990), hizo una primera propuesta de 18 esquemas divididos en 5 dimensiones, después de diferentes versiones psicométricas del cuestionario original, una de las que tuvo mayor aceptación por la consistencia y precisión en sus resultados, fue la creada en 1999 que evalúa 16 esquemas en una prueba que consta de 205 ítems llamada "Young Schema Questionaire Long Form- Second Edition”.

Esta versión fue validada en Colombia por Castrillón y colaboradores en el 2005 y en población peruana mediante el estudio León y Sucari en el 2012 que consta de 45 ítems que evalúa 11 esquemas mentales desadaptativos. Se realizó un análisis psicométrico en una muestra de dos colegios de Lima Metropolitana que dio como resultado una validez de contenido por el método de criterio de jueces, con puntajes $\mathrm{V}$ de Aiken de 0.97 y una confiabilidad interna con un Alfa de Cronbach de 0.9. El índice de consistencia interna oscilo en los diferentes esquemas entre 0.73 a 0.76 representando una confiabilidad de la prueba, identificando un buen nivel de confiabilidad en todos los componentes, el cual por su validez y confiabilidad aceptable ha sido utilizado por Cipriano (2017) para el estudio con estudiantes de 5to de secundaria en Trujillo -Perú y por Flores y Vilca (2017) en una investigación para conocer los esquemas mentales disfuncionales en 155 adolescentes arequipeños.

En el presente estudio se llevó a cabo un análisis factorial exploratorio (AFE) y con rotación varimax con un método de extracción de máxima verosimilitud; para la explicación del total de la varianza en base a los factores que arrojó el análisis, debido a que este minimiza el número de variables con cargas altas en alguno de los factores, mejorando así su interpretación. Posterior a ello se llevó a cabo un análisis de consistencia interna por medio del coeficiente de Alpha de Cronbach, sucesivo a ello se aplicó el mismo procedimiento para cada uno de los factores que se presentan en el instrumento.

\section{Validez}

En base al análisis factorial exploratorio, dio como resultado una medida de KMO de 0.983 y un nivel de significancia de 0.000 , lo cual refiere una alta correlación entre los ítems del instrumento de evaluación. El análisis factorial rotado varimax, muestra que el instrumento cuenta con 11 factores y 45 ítems finales. Estos 11 factores explican el $45.80 \%$ de la varianza. En la tabla $\mathrm{N}^{\circ} 4$ se muestran las varianzas explicadas de cada una de las escalas del instrumento y el número de ítems por cada uno:

Se hizo este análisis para poder indagar en si los 45 ítems y 11 escalas usadas de la versión (León \& Sucari, 2012) funcionaban de manera correcta e igual con la población evaluada en nuestro estudio. A partir de nuestros resultados del AFE, se observa que la prueba se comporta de manera adecuada 
en la población que hemos utilizado, debido a que se mantienen 45 ítems finales los cuales desembocan en 11 escalas.

Tabla 4

Análisis Varimax - Rotado

\begin{tabular}{llccc}
\hline $\mathbf{N}^{\circ}$ & \multicolumn{1}{c}{ Factor } & Número de ítems & \% de varianza & \% acumulado \\
\hline 1 & Privación Emocional & 5 & 19.85 & 19.85 \\
2 & Desconfianza & 5 & 4.43 & 24.28 \\
3 & Abandono & 6 & 4.50 & 28.77 \\
4 & Vulnerabilidad al Daño & 4 & 4.36 & 33.13 \\
5 & Entrampamiento & 2 & 2.92 & 36.05 \\
6 & Auto-sacrificio & 4 & 2.64 & 38.69 \\
7 & Inhibición Emocional & 3 & 1.97 & 40.66 \\
8 & Hipercritinismo & 3 & 1.85 & 42.50 \\
9 & Perfeccionismo & 4 & 1.17 & 43.67 \\
10 & Grandiosidad & 3 & 1.07 & 44.74 \\
11 & Autocontrol & 6 & 1.06 & 45.80 \\
\hline
\end{tabular}

\section{Confiabilidad}

Posterior al AFE, se procede a realizar el análisis de consistencia interna con el coeficiente de Alfa de Cronbach. Se observa que la prueba tiene una consistencia interna alta $(\alpha=.912)$ determinando así una alta confiabilidad. A su vez en la tabla $\mathrm{N}^{\mathrm{o}} 2$ se observa que por medio del análisis de cada uno de los factores y/o escalas que tiene la prueba, se encuentran entre los rangos de 0.70 y 0.83 de Alfa de Cronbach, lo que determina, que todas las escalas cuentan con una consistencia interna valida, es decir, son confiables.

\section{Tabla 5}

Análisis de Consistencia Interna - Factores

\begin{tabular}{llccc}
\hline & & Media (DT) & Percentiles (25-50-75) & $\boldsymbol{\alpha}$ \\
\hline 1 & Privación Emocional & $15.00(5.41)$ & $11-15-19$ & 0.70 \\
2 & Desconfianza/Abuso & $17.85(5.45)$ & $14-18-22$ & 0.83 \\
3 & Abandono & $16.95(6.96)$ & $12-16-22$ & 0.72 \\
4 & Vulnerabilidad al daño & $17.85(5.29)$ & $10-14-18$ & 0.77 \\
5 & Entrampamiento & $5.57(2.52)$ & $4-6-7$ & 0.73 \\
6 & Autosacrificio & $15.14(4.96)$ & $12-15-19$ & 0.76 \\
7 & Inhibición Emocional & $10.13(3.89)$ & $7-10-13$ & 0.74 \\
8 & Hipercritinismo & $12.72(4.46)$ & $10-12-16$ & 0.73 \\
9 & Perfeccionismo & $11.01(3.45)$ & $9-11-13$ & 0.70 \\
10 & Grandiosidad & $8.92(3.19)$ & $6-9-11$ & 0.72 \\
11 & Autocontrol & $18.58(6.32)$ & $14-18-23$ & 0.77 \\
\hline
\end{tabular}


Posterior al debido análisis de consistencia interna, se procedió al análisis de percentiles para determinar los baremos de cada uno de los factores en base a tres niveles: bajo, medio y alto.

\section{RESULTADOS}

En base al análisis de asimetría - curtosis, Kolmogorov - Smirnov y Shapiro Wilk se determina que los valores están dentro de la normalidad y se procede a hacer un análisis de datos de forma paramétrica.

En la tabla $\mathrm{N}^{0} 6$ se observan las diferencias significativas en relación a estructura familiar en base a los esquemas mentales; en la cual se observa diferencias significativas por medio de la prueba ANOVA en los esquemas mentales desadaptativos de: privación emocional $(\mathrm{F}=3.804, \mathrm{p}=0.01)$, desconfianza $(\mathrm{F}=2.880$, $\mathrm{p}=0.02)$, hipercritinismo $(\mathrm{F}=2.683, \mathrm{p}=0.03)$ y grandiosidad $(\mathrm{F}=2.391, \mathrm{p}=0.05)$.

\section{Tabla 6}

Prueba de ANOVA-Esquemas Mentales desadaptativos/Estructura familiar

\begin{tabular}{|c|c|c|c|c|c|c|c|}
\hline & & $\mathbf{N}$ & Media & $\begin{array}{l}\text { Desviación } \\
\text { estándar }\end{array}$ & $\mathbf{F}$ & Sig. & d \\
\hline \multirow{5}{*}{$\begin{array}{l}\text { Privación } \\
\text { Emocional }\end{array}$} & Biparental & 349 & 14.40 & 5.21 & \multirow{5}{*}{3.804} & \multirow{5}{*}{0.01} & \multirow{5}{*}{0.26} \\
\hline & Extensa & 93 & 15.18 & 6.02 & & & \\
\hline & Reconstruida & 42 & 16.36 & 5.29 & & & \\
\hline & Monoparental & 179 & 15.23 & 5.24 & & & \\
\hline & Sin núcleo & 50 & 17.10 & 5.66 & & & \\
\hline \multirow{5}{*}{ Desconfianza } & Biparental & 349 & 17.58 & 5.32 & \multirow{5}{*}{2.880} & \multirow{5}{*}{0.02} & \multirow{5}{*}{0.20} \\
\hline & Extensa & 93 & 17.55 & 5.60 & & & \\
\hline & Reconstruida & 42 & 20.14 & 4.85 & & & \\
\hline & Monoparental & 179 & 17.96 & 5.37 & & & \\
\hline & Sin núcleo & 50 & 19.12 & 5.81 & & & \\
\hline \multirow{5}{*}{ Hipercritinismo } & Biparental & 349 & 12.62 & 4.54 & \multirow{5}{*}{2.683} & \multirow{5}{*}{0.03} & \multirow{5}{*}{0.22} \\
\hline & Extensa & 93 & 12.44 & 4.51 & & & \\
\hline & Reconstruida & 42 & 14.24 & 3.92 & & & \\
\hline & Monoparental & 179 & 12.31 & 4.24 & & & \\
\hline & Sin núcleo & 50 & 13.94 & 4.67 & & & \\
\hline \multirow{5}{*}{ Grandiosidad } & Biparental & 349 & 8.72 & 3.23 & \multirow{5}{*}{2.391} & \multirow{5}{*}{0.05} & \multirow{5}{*}{0.29} \\
\hline & Extensa & 93 & 8.58 & 3.51 & & & \\
\hline & Reconstruida & 42 & 9.98 & 2.82 & & & \\
\hline & Monoparental & 179 & 8.93 & 3.03 & & & \\
\hline & Sin núcleo & 50 & 9.64 & 2.99 & & & \\
\hline
\end{tabular}


Siendo así que se observan mayores niveles de esquemas mentales desadaptativos en la estructura familiar reconstruida, en comparación con las otras estructuras consideradas. A su vez, se observan en la tabla $\mathrm{N}^{0} 6$ que el tamaño de efecto en las diferencias significativas es bajo en cada una de ellas, por lo cual no vendría a ser una diferencia marcada aun con presencia de significancia, ya que estas son muy leves entre uno y otro grupo y no se puede hablar de llevar esta diferencia a otras poblaciones con las mismas características.

\section{DISCUSIÓN}

Estudios demuestran que la composición y dinámica familiar, son factores clave para predecir algunos el comportamiento de los miembros del hogar (Cano et al., 2016; Cabrera et al., 2006; Belsky, 2005). Dado que, por lo general el concepto de composición familiar se entiende en términos de integrantes y miembros que la componen, y las dinámicas relacionales se aproximan a la funcionalidad como lo plantea la teoría sistémica (Prada, 2013; Espinal et al., 2006), es necesario no separar estas dos perspectivas a la hora de abordar a la familia como una necesidad connatural del individuo (Vargas, 2014; Martínez et al., 2013; Coleman, 2013).

Nathan Ackerman (1966), plantea desde una perspectiva psicológica, que la familia debe ser un escenario en el que se construyan personas que experimenten un bienestar integral y en el que se versen criterios como la satisfacción de las necesidades físicas y emocionales, promoción de relaciones interpersonales fructuosas y un sistema de valores que dirija a todos sus miembros a la búsqueda de su autonomía y autorrealización (Haskins, 2015; Valladares, 2008; Del Río et al., 2004).

La literatura que analiza conjuntamente los patrones de pensamiento desadaptativos y la estructura familiares es limitada (Gantiva et al., 2009; Pera \& Jiménez, 2009); no obstante, los EMD han sido fundamento para explicar diferentes anomalías de la personalidad y comportamiento (Chuquimajo, 2014; Bárcenas, 2010). En ese sentido, según la teoría expuesta y estudios de corte sociológico y psicológico, la familia destacaría como el terreno de origen de los esquemas mentales funcionales y disfuncionales (Mayorga et al., 2016).

Nos propusimos en este estudio conocer la presencia de los EMD en las diferentes estructuras familiares vistas hoy en día. Diferentes autores respaldan a las familias nucleares biparentales como el contexto más saludable y favorable para el desarrollo psicológico y social de los hijos (Potter, 2010; Wood, Repetti \& Roesch, 2004); esto se podría deber a que en los hogares biparentales, la relación de pareja y de padres e hijos, al ser constitutivamente vinculantes generarían el sentido propio de lo que se llama familia (Wilcox, 2017; Donati, 2013; Del Valle, 2004); por lo tanto, serían capaces de prever considerablemente la salud psicológica de todos sus miembros (Shapiro et al, 2000; Rogers \& White, 1998), resaltando 
el quehacer significativo de ambos padres (Brown et al., 2016; Olhaberry \& Santelices, 2013).

Si los esquemas mentales disfuncionales tienen como origen las consecuencias de las relaciones difusas más significativas de los contextos más representativos (Iraugi, Martínez, Iriarte \& Sanz, 2011), se podría sugerir que los cambios en la configuración de los subsistemas familiares modificaría los vínculos, roles y funciones de sus miembros (Belsky, 2005; Cortés, Cantón \& Cantón, 2011), de manera que la presente investigación abre luces para analizar de qué manera la composición familiar podría estar asociada al desarrollo de un comportamiento insano.

Ya la literatura confiere a los EMD un origen en la familia; en ese sentido, nuestros resultados sugieren que el EMD de privación emocional se activa dentro de los hogares reconstruidos conformados por nuevos integrantes en el hogar, espacios que carecen de jerarquía de subsistemas parental y filial, por la carencia de figuras permanentes y estables que solidifiquen este sentimiento de pertenencia, seguridad y estabilidad personal (Pliego \& Castro, 2015; Del Valle, 2004). El segundo EMD encontrado en los hogares reconstruidos fue el grandiosidad o necesidad de hacerse notar y ser importante para los demás miembros del hogar, que no poseen de forma innata un vínculo paternal o fraternal de entrega y amor, sino que tendría que construirse con el paso del tiempo y esto podría resultar en una búsqueda de reconocimiento, admiración carente de reciprocidad y empatía con los demás a consecuencia de una posible vulneración de roles o jerarquía (Cortés et al., 2011).

De la misma forma el EMD de desconfianza parte de la premisa de que cualquier tipo de acercamiento personal tiene como fin un interés perjudicial dirigido a herir, lastimar, sacar provecho y traicionar; los presenten hallazgos dilucidaron que este esquema se encuentra más inclinado a presentarse en hogares reconstruidos y esto podría darse porque estos hogares parten del resquebrajamiento de una unidad para pasar a otra con diferente dinámica, funcionalidad y que podría provocar sentimientos poco reconfortantes en cuanto a la protección y confianza que requiere un adolescente en el núcleo familiar (Wilcox, 2017; Roca, 2010).

Los estilos de vida propuestos en las nuevas tendencias de hogar suelen conducir a una heterogeneidad, lo que provocaría señalando a Berger y Luckmann (1997) nuevas formas y supondría el debilitamiento de las funciones, normas, valores, entre otros elementos que darían a lugar a una desinstitucionalización de la familia, lo que podría responder al EMD de hipercritinismo o inconformidad hacia la propia persona por sus propios valores y sentido de pertenencia presentado con mayor relevancia en los hogares reconstruidos, en los que se carecería de una 
dirección compacta y estable que regule de manera positiva el desarrollo de sus miembros hacia un horizonte reconciliador y de auto aceptación personal.

Ante lo dicho Baumann (2010), advierte que si la familia se vuelve líquida, la sociedad también, en la misma línea Donati, (2013); menciona que al ser la familia un bien relacional primario, requiere una solidez propia de su esencia, ya que de ella depende la realización de la humanidad (Hanskins, 2015; ODSA, 2014). En este sentido, el institucionalizar nuevas funciones y relaciones producirían patterns de comportamiento que tenderían a permanecer a lo largo del tiempo; solo a partir de pautas sólidas se posibilitaría la formación de hábitos, normas y valores, que son indispensables en el desarrollo integral de los hijos y bienestar de la pareja (Cuddeback, 2017; Chong, 2016).

\section{CONCLUSIONES}

El presente estudio concluye que la estructura familiar reconstruida es escenario de la formación de algunos esquemas mentales desadaptativos como los de privación emocional, desconfianza, hipercritinismo y grandiosidad. En ese sentido la composición familiar también es un elemento del cual se desprenderían diferentes comportamientos que afectan de manera positiva o negativa a la salud psicológica del adolescente.

\section{NOTA DE RECONOCIMIENTO}

El siguiente estudio estuvo financiado por el Instituto para el Matrimonio y la Familia y la Dirección de investigación de la Universidad Católica San Pablo. El primer autor forma parte del equipo de investigación del Instituto para el Matrimonio y la Familia y los coautores forman parte de la escuela profesional de Psicología.

\section{REFERENCIAS}

Ackerman, N. (1966). Diagnóstico y tratamiento de las relaciones familiares. 2da Ed. Ediciones Hormé: Buenos Aires.

Bahamón, M. (2013). Relación entre esquemas inadaptativos, distorsiones cognitivas y síntomas de ludopatía en jugadores de casinos. Pensamiento Psicológico, 2 (11), 89102. https://bit.ly/2NVaBKh

Bárcenas, K. (2010). Familias diversas: de la institución al movimiento. Estructuras y dinámicas en la reconfiguración del orden. (Tesis de maestría). Instituto Tecnológico y de Estudios Superiores de Occidente. Jalisco. https://bit.ly/2NXwZTq

Bárcenas, K. (2012). Las familias mexicanas: de la institución al movimiento. Trayectorias, significados e imaginarios en la configuración de la diversidad familiar. Sociedade e Cultura, 15 (2), 263-274. https://bit.ly/2NrMw0P

Bauman, Z. (2010). Amor Líquido. Acerca de la fragilidad de los vínculos Humanos. Buenos Aires: Fondo de Cultura Económica. 
Bertalnffy, L. (1968). Teoría general del sistema: ensayos sobre su fundamento y desarrollo. Nueva York: George Braziller.

Belsky, J. (2005). Family Influences on psychological development. Psyquiatry, 4 (7): 41 44. https://doi.org/10.1383/psyt.2005.4.7.41

Berger, P. y Luckmann, T. (1997). Modernidad, pluralismo y crisis de sentido. Barcelona: Ediciones Paidós.

Bronfenbrenner, U. (1979). La ecología del desarrollo humano. Barcelona: Paidós.

Brown, S., Mainning, W. \& Payne, K. (2016). Family structure and children's economic well-being: incorporating same-sex cohabiting mother families. Population Research \& Policy Review, 35, 1- 21. https://bit.ly/2wPRjzo

Cabrera, V., Guevara, I. y Barrera, F. (2006). Relaciones paternales y su influencia en el ajuste psicológico de los hijos. Acta Colombia de Psicología, 9 (2), 115- 126. https:// bit.ly/2wZPZKL

Cano, A., Motta, M., Valderrama, L. y Gil, C. (2016). Jefatura masculina en hogares monoparentales: adaptaciones de los hombres a las necesidades de sus hijos. Revista Colombiana de ciencias sociales, 39 (1), 123-145. https://doi.org/10.15446/rcs. v39n1.56344

Cantón, J., Cortés, M del R. y Justicia, M.D. (2002). Las consecuencias del divorcio en los hijos. Psicopatología Clínica, Legal y Forense, 2 (3), 47- 66. https://bit.ly/2MedODm

Castrillón, D; Chávez, L; Ferrer, A; Londoño, N; Maestre, K; Marín, C y Schniter, M. (2005). Validación del Young Schema Questionaire Long Form - Second Edition (YSQ -L2) en población Colombiana. Revista Latinoamericana de psicología, 37 (2), 541- 560. https://bit.ly/2wSUJAP

Castro, M., López, A., \& Sueiro, E. (2009). Perfil sociodemográfico - penal y distorsiones cognitivas en delincuentes sexuales. Revista Galego Portuguesa de psicoloxia e educación, 17 (1,2), 155- 166. https://bit.ly/2M68AJv

Cavanagh, S., Krissey, S. \& Raley, R. (2008). Family structure history and adolescent romance. Journal of Marriage and Family, 70, 698-714. https://bit.ly/2MVVaVS

Cepeda, Z. y Ruiz, J.I. (2016). Distorsiones cognitivas: diferencias entre abusadores sexuales, delincuentes violentos y un grupo control. Revista Criminalidad, 58 (2), 141-156. https://bit.ly/2wVJto5

Cipriano, D. (2017). Esquemas desadaptativos tempranos y dependencia emocional en estudiantes de 5to año de secundaria de las Instituciones Educativas. (Tesis de licenciatura). Universidad César Vallejo. Chimbote. https://bit.ly/2MasEug

Chávez, D. (2017). Propiedades psicométricas del Cuestionario de Esquemas Maladaptativos de Young en adolescentes de Otuzco. (Tesis de licenciatura). Universidad César Vallejo. Trujillo. https://bit.ly/2wPTbrU

Chong, A. \& Mickelson, K. (2016). Perceived fairness and relationship satisfaction during the transition to parenthood: The Mediating Role of Spousal Support, 37 (1), 3 - 28. https://doi.org/10.1177/0192513X13516764 
Chuquimajo, S. (2014). Personalidad y Clima Social Familiar en adolescentes de Familia Nuclear Biparental y Monoparental (Tesis de Maestría). Universidad Nacional Mayor de San Marcos. Lima. https://bit.ly/2NsAcNN

Coleman, C. (2013). Partnership in Europe; its variety, trends and dissolution. Finnish Yearbook of Population Research, 48, 5- 49. https://bit.ly/2Cs7ZT3

Cortés, M del R., Cantón, J. y Cantón, D. (2011). Estructura del hogar y conflictividad entre los padres. International Journal of Developmental and Educational Psychology, 1 (2), 503- 510. https://bit.ly/2M8yQTN

Cuddeback, J. (2017). Reclaiming a Father's Presence at Home. [Mensaje en un blog] Institute for Family Studies. Recuperado de https://bit.ly/2MZJQbv

De la Vega, R. y Gómez, G. (2012). Intervención cognitivo-conductual en conductas alimentarias de riesgo. Psicología y Salud, 22 (2), 225- 234. https://bit.ly/2NXhlY2

Del Río, Barrera, A.M. y Vargas, E. (2004). Factores de las relaciones padres-hijos que se asocian con las expectativas de vinculación y de apoyo a la autonomía que tienen los adolescentes de las relaciones románticas. Suma psicológica, 11 (1), 95-110. https:// bit.ly/2NwTT7n

Del Valle, A. (2004). El futuro de la familia: La familia. Revista de Pensamiento Cristiano, 17, 9- 26. https://bit.ly/2sov5BF

Donati, P. (2013). La familia como raíz de la sociedad. Madrid: Biblioteca de autores cristianos.

Espinal, I., Gimemo, A. y Gonzáles, F. (2006). El enfoque sistémico en los estudios sobre la familia. Revista internacional de sistemas, 21-34. https://bit.ly/2oNH1N2

Estévez, A. y Calvete, E. (2007). Esquemas cognitivos en personas con conducta de juego patológico y su relación con experiencias de crianza. Clínica y Salud, 18 (1), 23- 43. https://bit.ly/2oLRlEi

Ferrer, R. Peña, A. Gómez, N. y Pérez, K. (2009). Esquemas maladaptativos tempranos en pacientes diagnosticados con cáncer atendidos en tres centros oncológicos de la ciudad de Santa Marta. Psicología desde el Caribe, 24: 180- 2014. https://bit. $1 y / 2 o P O s S K$

Flores, M. y Vilca, M. (2017). Esquemas desadaptativos tempranos en adolescentes con problemas de conducta. (Tesis de licenciatura). Universidad Nacional de San Agustín. Arequipa. https://bit.ly/2oO9Ojr

Gantiva, C., Bello, J., Vanegas, E. y Sastoque, Y. (2009) Historia de maltrato físico en la infancia y esquemas maladaptativos tempranos en estudiantes universitarios. Acta Colombiana de psicología, 12 (2), 127- 134. https://bit.ly/2M6az0p

Haskins, R. (2015). The family is here to stay or not. The Future of Children, 25 (2), 129153. https://bit.ly/2QcJ1d2

Hernández, R., Fernandez, C. y Baptista, P. (2014). Metodología de la investigación. México D.F: The McGraw-Hil 
Huarcaya, G. (2011). La familia peruana en el contexto global. Impacto de la estructura familiar y la natalidad en la economía y el mercado. Mercurio Peruano: Revista de humanidades, 524, 13-21. https://bit.ly/2Me17Zn

IBM Corp. Released (2013). IBM SPSS Statistics for Windows, Version 24.0. Armonk, NY: IBM Corp.

Iraugi, L., Martínez, A., Iriarte, L., y Sanz, M. (2011). Modelo cognitivo-contextual del conflicto interparental y la adaptación de los hijos. Anales de psicología, 27 (2), $562-$ 573. https://bit.ly/2CEheQi

Lemos, M., Londoño, N y Zapata, J. (2007). Distorsiones cognitivas en personas con dependencia emocional. Informes psicológicos, 9, 55- 69. https://bit.ly/2CsgzBf

León, A., Salazar, A. y Puerta, D. (2013). Esquemas maladaptativos tempranos en sujetos con organización de significado personal depresiva. Katharsis, 16, 99- 120. https:// doi.org/10.25057/issn.2500-5731

León, K y Sucari, C. (2012). Adaptación del cuestionario de esquemas de Young en adolescentes de dos distritos de Lima Sur. (tesis de licenciatura) Unife: Lima. https:// bit.ly/2MXd7U6

Martínez, M., Robles, C., Utria, L. y Amar, J. (2014). Legitimación de la violencia en la infancia: un abordaje desde el enfoque ecológico de Bronfenbrenner. Psicología desde el Caribe, 31 (1), 133-160. https://bit.ly/2MXhpdV

Martínez, M.C., Estévez, E. \& Inglés, C. (2013). Diversidad familiar y ajuste psicosocial en la sociedad actual. Revista Psicología. com, 17 (6), 1- 22. https://bit.ly/2wPY3O4

Mayorga, C., Godoy, M.P., Riquelme, S. y Ketterer, L. (2016). Relación entre problemas de conducta en adolescentes y conflicto interparental en familias intactas y monoparentales. Revista Colombiana de Psicología, 25 (1), 107-122. http://dx.doi. org/10.15446/rcp.v25n1.48705

Ministerio de Educación del Perú (MINEDU). (2017). Servicios educativos. Lima: Unidad de estadística educativa. Recuperado de http://escale.minedu.gob.pe/inicio

Múnera, J. y Tamayo, D. (2013). Esquemas maladaptativos tempranos en mujeres víctimas de violencia intrafamiliar, usuarias del C.A.V (Centro de atención a víctimas), fiscalía, seccional Medellín. Revista Psicoespacios, 7 (10), 186- 202. https://bit.ly/2NTFT4g

Observatorio de la Deuda Social Argentina (ODSA). (2014). Los argentinos y la familia. Buenos Aires: Universidad Católica Argentina. https://bit.ly/2M8xsQM

Olhaberry, M. y Santelices, M.P. (2013). Presencia del padre y calidad de la interacción madre-hijo: un estudio comparativo en familias chilenas nucleares y monoparentales. Universitas Psychologica, 12 (3), 833-843. http://dx.doi.org/10.11144/Javeriana. UPSY12-3.ppci

Olhaberry, M. y Farkas, Ch. (2012). Estrés materno y configuración familiar: estudio comparativo en familias chilenas monoparentales y nucleares de bajos ingresos. Universitas Psychologica, 11 (4), 1317-1326. https://bit.ly/2MaPNwZ. 
Palacios, M.J. y Rodrigo. J. (1998). Familia y desarrollo humano. Madrid: Alianza Editorial.

Parsons, T. y Bales R.F. (1955). Family, socialization and interaction process. Glencoe: The Free Press.

Perea, M. C. y Jiménez, M. A. (2009). Estudio comparativo de escolares murcianos, hijos de padres separados y de familias intactas. Familia, 39, 105-122. https://bit. $\mathrm{ly} / 2 \mathrm{CvgCwb}$

Pliego, F. y Castro, R. (2015). Tipos de familia y Bienestar de niños y adultos. El debate del siglo XXI en 13 países democráticos. Arequipa: Fondo Editorial UCSP.

Potter, D. (2010). Psychosocial well-being and the relationship between divorce and children's academic achievement. Journal of Marriage and Family, 72 (4), 933-946. http://dx.doi.org/10.1111/j.1741-3737.2010.00740.x

Prada, S. (2013). Corrientes cognitivas y sistémicas en psicoterapia: Integración y paradigma. Reflexiones, 92 (2), 155-161. https://bit.ly/2wZWdu7

Roca, N. (2010). El proceso de desinstitucionalización de la vida familiar: La maternidad/ paternidad en solitario por opción en España. Documento presentado en el $\mathrm{X}$ Congreso Español de Sociología: "Treinta años de sociedad, treinta años de sociología”. Pamplona 1 de Julio del 2010. https://bit.ly/2CDmdQS

Rodríguez, E. (2009). La terapia centrada en esquemas de Jeffrey Young. Revista Avances de psicología, 17 (2), 59- 74. https://bit.ly/2M8qTxN

Rodríguez, I. y Menéndez, S. (2003). Los retos de las nuevas realidades familiares. Portularia, 3, 9- 32. https://bit.ly/2CxtNg4

Rogers, S.J., \& White, L.K. (1998). Satisfaction with parenting: the role of marital happiness, family structure, and parent's gender. Journal of Marriage and the family, 60, 293 - 208. http://dx.doi.org/1010.2307/353849

Shapiro, A., Gottman, J. \& Carrére, S. (2000). The baby and the marriage. Identifying factors that buffer against decline in marital satisfaction after the first baby arrives. Journal of Family Psychology, 14 (1), 59- 70. http://dx.doi.org/10.1037//08933200.14.1.59

Stock, L., Corlyon, J., Castellanos, S., \& Gieve, M. (2014). Personal relationships and poverty an evidence and policy review. London: The Tavistock Institute. https://bit. ly/2Q71FFT

Valladares, A. (2008). La familia. Una mirada desde la psicología. Revista Electrónica de las Ciencias Médicas en Cienfuegos, 6 (1), 4 - 12. https://bit.ly/2NRXh9A

Vargas, H. (2014). Tipo de familia, ansiedad y depresión. Revista Médica Herediana, 25, 57-59. https://bit.ly/2NWhY4m

Vite, A., Negrete, A. y Miranda, D. (2012). Relación mediacional de los esquemas cognitivos maternos en los problemas de comportamiento infantil. Psicología y Salud, 22 (1), 27- 36. https://bit.ly/2MayVpO 
Wilcox, B. (2017). Married parents: One way of reducing child poverty. [Mensaje en un blog]. AEI. Recuperado de https://bit.ly/2CskQoh

Wood, J., Repetti, R. L. \& Roesch, S. C. (2004). Divorce and children's adjustment problems at home and school: The role of depressive/withdrawn parenting. Child Psychiatry and Human Development, 35(2), 121-142. https://doi.org/10.1007/s10578004-1881-6

World Family Map. (2017). Mapping family change and child Well-Being Outcomes. New York - Barcelona: Social Trends Institute. https://bit.ly/2M4OEH6

Young, J., Klosko, J. \& Weishaar, M. (2013). Schema Therapy. A practitioner's guide. New York: Guilford Publications 
